\title{
Mantle Cell Lymphoma with Histological Progression to Pleomorphic Lymphoma in Two Cattle
}

\author{
Akiyo HAGIWARA ${ }^{1}$, Morihiro SAITO ${ }^{2}$, \\ Yoshiharu ISHIKAWA ${ }^{3}$ and Koichi KADOTA ${ }^{3 *}$ \\ ${ }^{1}$ Saitama Prefecture Meat Inspection Center (Saitama, Saitama 338-0001, Japan) \\ ${ }^{2}$ College of Nutritional Science, Kagawa Nutrition University (Sakado, Saitama 350-0288, Japan) \\ ${ }^{3}$ Hokkaido Research Station, National Institute of Animal Health, National Agriculture and Food \\ Research Organization (Sapporo, Hokkaido 062-0045, Japan)
}

\begin{abstract}
An abattoir survey for bovine enzootic leukosis revealed that severe splenomegaly and hepatomegaly were observed in a heifer (case 1) and a steer (case 2), respectively, in addition to great enlargement of certain lymph nodes. Both cases tested positive for bovine leukemia virus (BLV) by polymerase chain reaction. Histologically, the neoplastic tissues in case 1 were characterized by a mantle zone growth pattern of medium-sized cells showing a transition to highly pleomorphic and atypical cells. Similar histological characteristics were also observed in case 2, but neoplastic mantle cells forming focal lesions were more pleomorphic than in case 1. B cell markers CD20 and CD79a were expressed in cases 1 and 2, as well as CD5 and cyclin D1, which are important markers for human mantle cell lymphoma. Cases of pleomorphic B cell lymphoma with large abdominal tumor masses are thought to be derived from body cavity B-1 cells, whereas this study suggests that nodal pleomorphic B cell lymphoma is of mantle cell origin.

Discipline: Animal health

Additional key words: Bovine leukemia virus, cyclin D1, immunohistochemistry
\end{abstract}

\section{Introduction}

In the World Health Organization (WHO) classification of human lymphoid neoplasms, mantle cell lymphoma is a monomorphic lymphoid proliferation with a mantle zone, follicular, vaguely nodular or diffuse growth pattern (Swerdlow et al. 2008). This lymphoma derived from lymphocytes in the primary follicles or mantle zones of secondary follicles is characterized by the expression of CD5 and cyclin D1 (Swerdlow et al. 2008), but is usually negative for cytoplasmic immunoglobulin (cIg) (Feller \& Diebold 2004). In the blastoid and pleomorphic variants of this lymphoma, the tumor cells are lymphoblastoid or more pleomorphic and mitotically active than in classical mantle cell lymphoma (Feller \& Diebold 2004, Swerdlow et al. 2008). Despite the lack of immunophenotypic evidence, mantle cell lymphoma has been diagnosed based on the growth pattern in dogs (Valli et al. 2006).

In cattle, most lymphomas associated with bovine leukemia virus (BLV) are characterized by cytological pleomorphism and atypia, and are designated pleomorphic
B cell lymphomas (Kagawa et al. 2009, Murayama et al. 2011, Iwama et al. 2013, Hagiwara et al. 2014). Some cases are macroscopically characterized by abdominal tumor masses and considered to be derived from B-1 cells in the abdominal cavity (Abe et al. 2007, Hagiwara et al. 2014). The current study reports two cases of CD5- and cyclin D1-positive nodal mantle cell lymphoma where histological progression to pleomorphic B cell lymphoma was observed.

\section{Materials and methods}

\section{Animals}

A crossbred (Japanese Black $\times$ Holstein) heifer (case 1) and a Japanese Black steer (case 2), both aged 27 months, appeared healthy just before slaughter.

\section{Genetic detection for BLV}

DNA obtained from a tumor nodule on the epicardium of the atrial appendage (case 1) and from blood (case 2) was analyzed by quantitative real-time polymerase chain 
reaction (PCR) for BLV (Somura et al. 2014).

\section{Histological and immunohistochemical examinations}

Tissue samples were fixed in 10\% neutral-buffered formalin, embedded in paraffin, sectioned at $4 \mu \mathrm{m}$, and stained with hematoxylin and eosin (HE). Selected sections were stained for immunohistochemistry using the streptavidin-biotin complex/horseradish peroxidase (SAB-PO) method with a commercially available Histofine SAB-PO kit (Nichirei, Tokyo, Japan). The primary antibodies utilized were rabbit polyclonals to human CD20 (prediluted; Spring Bioscience, Pleasanton, CA, USA), human $\kappa$ light chain, human $\lambda$ light chain (prediluted; Fitzgerald, Acton, MA, USA), human CD5 (1:400; Pierce Biotechnology, Rockford, IL, USA), human CD3e (1:50; Dako A/S, Glostrup, Denmark) and human cyclin D1 (prediluted; Lab Vision, Fremont, CA, USA), and mouse monoclonals to human CD79a, HM57 (1:25; Dako A/S) and proliferating cell nuclear antigen (PCNA), PC10 (prediluted; BioGenex, San Ramon, CA, USA). Antigen retrieval was by enzymatic digestion with $0.05 \%$ pepsin at $37^{\circ} \mathrm{C}$ for 25 minutes $(\mathrm{CD} 3 \varepsilon)$, or microwave heating in $10 \mathrm{mM}$ citrate buffer, $\mathrm{pH} 6.0$ at $90^{\circ} \mathrm{C}$ for 9 minutes (CD5, cyclin D1, CD79a, PCNA). Antibodies were detected by incubation with 3,3'-diaminobenzidine tetrahydrochloride solution. Sections were counterstained with hematoxylin for microscopic examination.

\section{Results}

\section{Gross pathology}

In case 1, the spleen was extremely enlarged with very large lymphoid follicles and elevation of cut surfaces. The superficial cervical, subiliac, popliteal, ruminal and internal iliac lymph nodes were greatly enlarged, as was the lymph node of the right paralumbar fossa. The bronchial lymph nodes and some superficial and abdominal nodes were less severely affected. Their cut surfaces were milky white and homogeneous occasionally with hemorrhage. There were nodules up to $1 \mathrm{~cm}$ in diameter, sparsely distributed on the right parietal pleura, and a $3 \times 4 \mathrm{~cm}$ nodule on the pancreas. A tumor nodule was seen on the epicardium of the atrial appendage.

In case 2, the liver was highly enlarged with pallor and hard consistency, but no abnormal findings were detected in the spleen. The mediastinal, bronchial and subiliac lymph nodes were greatly enlarged, and the internal iliac lymph nodes and such superficial nodes as the mandibular, superficial cervical and popliteal were moderately enlarged. These swollen lymph nodes were milky white with a homogeneous amorphous appearance on the cut section. Regional lymph nodes of the liver and stomach appeared normal. There were several nodules up to $5 \mathrm{~cm}$ across just beneath the thoracic cutaneous muscle. Multiple nodules up to $1 \mathrm{~cm}$ in diameter were observed on the rectal serosa, and a few similar nodules were present on the abomasal mucosa.

\section{Genetic detection for BLV}

The BLV copy number was 727 copies/100 ng DNA in case 1 , and 2,690 in case 2 .

\section{Histological and immunohistochemical findings}

Neoplastic growths were observed mostly in macroscopically visible lesions in both cases. In case 1 , the majority of the splenic tissue was displaced by neoplastic mantle cells, which showed a focal (mantle zone, follicular or vaguely nodular), sheet-like or diffuse growth pattern. The popliteal, mediastinal and ruminal lymph nodes were composed almost exclusively of sheet-like or diffuse growth of neoplastic mantle cells, but in places the cells were admixed with larger cells. In contrast, atypical and highly pleomorphic cells predominated in the superficial cervical, subiliac, internal iliac and mesenteric lymph nodes, and the mantle cell component partially remained (Fig. 1A). In the other nodes examined, neoplastic mantle cells showed variable patterns of focal growth (Fig. 1B), and atypical and highly pleomorphic cells were observed around or between these foci (Fig. 1C). A few focal lesions of neoplastic mantle cells were observed in the lungs, and similar cells were very numerous within blood vessels. Some lymphatic follicles were replaced by neoplastic mantle cells in the small intestine. Several neoplastic mantle cells were observed in the epicardial nodule detected macroscopically.

In case 2 , the mediastinal and bronchial lymph nodes revealed a follicular or vaguely nodular growth pattern, and highly pleomorphic cells were inconspicuous. Focal or sheet-like growth of neoplastic mantle cells and diffusely growing pleomorphic cells were seen in the other lymph nodes examined. In the liver, Glisson's sheath was moderately invaded by neoplastic mantle cells. The lungs were heavily infiltrated by neoplastic mantle cells.

Cytologically, the neoplastic mantle cells in case 1 were moderate in size, and had round, ovoid or slightly irregular nuclei and relatively abundant cytoplasm. The nucleoli were small to medium-sized, and the chromatin was slightly condensed (Fig. 1C). Larger cells with or without prominent nucleoli were rarely admixed with the predominant cells, and their nuclei were not very irregular. In contrast, pleomorphic cells were large or very large in size with variously sized nucleoli and moderately condensed chromatin, and occasionally with highly irregular or bizarre nuclei (Fig. 1D). Mitotic figures sometimes occurred in the mantle cells, but more frequently in the pleomorphic cells. In case 2, the neoplastic mantle cells were more variable in size and nuclear shape than in case 1 (Fig. 1E). The diffusely growing pleomorphic cells with irregular nuclear 

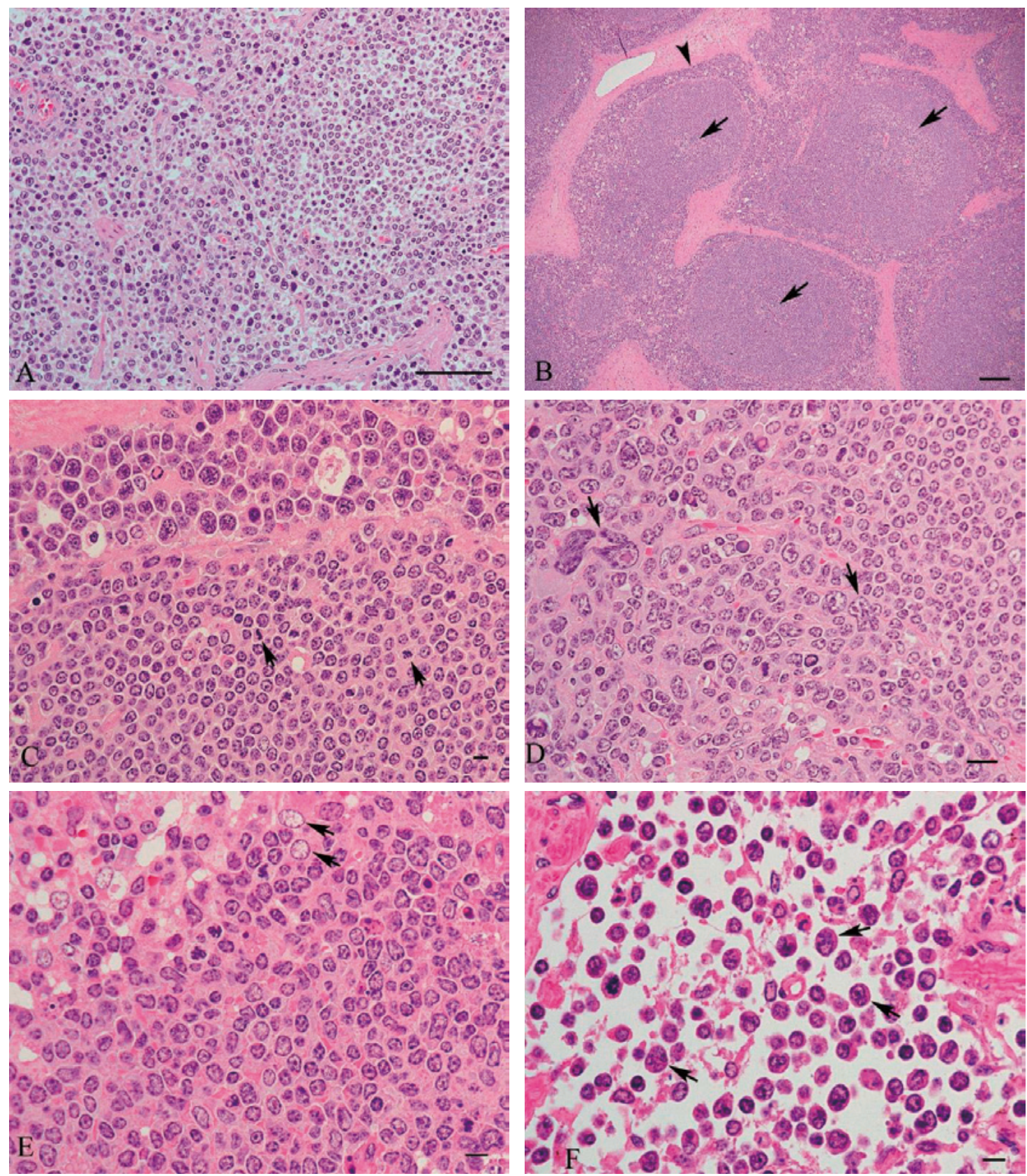

Fig. 1A. Case 1, superficial cervical lymph node

The architecture is mostly replaced by diffusely growing large pleomorphic cells, but an accumulation of neoplastic mantle cells is visible in this field (upper right). [HE; Bar $=50 \mu \mathrm{m}]$

Fig. 1B. Case 1, lymph node of the paralumbar fossa

Mantle cell foci with atrophic or hyperplastic follicle centers (arrows) are encircled by bands of pleomorphic cells in lymphatic sinuses (arrowhead), in which scattered macrophages impart a starry sky appearance. [HE; Bar $=100 \mu \mathrm{m}$ ]

Fig. 1C. Case 1, lymph node of the paralumbar fossa

Enlargement of the portion indicated by an arrowhead in Fig. 1B. Neoplastic mantle cells and larger and highly pleomorphic cells are visible, and mitotic figures of the former predominate in this field (arrows). [HE; Bar $=5 \mu \mathrm{m}]$

Fig. 1D. Case 1, lymph node of the paralumbar fossa

No distinct boundary is noted between areas of pleomorphic cells and neoplastic mantle cells (right). Arrows indicate giant pleomorphic cells with highly irregular bizarre nuclei. [HE; Bar $=10 \mu \mathrm{m}]$

Fig. 1E. Case 2, mediastinal lymph node

Neoplastic mantle cells are more variable in cell, nuclear and nucleolar size than in case 1. A residual follicle center with follicular dendritic cells (arrows) is visible at the upper left. [HE; Bar $=5 \mu \mathrm{m}$ ]

Fig. 1F. Case 2, superficial cervical lymph node

Pleomorphic cells with irregular or lobulated nuclei (arrows) are observed within a dilated lymphatic sinus. [HE; Bar $=5 \mu \mathrm{m}]$ 
contours varied in size (Fig. 1F), but unlike in case 1, giant cells were absent. Mitosis was occasionally observed in the mantle and pleomorphic cells.

Immunohistochemically, both types of lymphoma cells in cases 1 and 2 were positive for CD20 (Fig. 2A), CD79a and CD5, but not for CD3. Lymphoma cells expressing cIg were absent. Occasional neoplastic mantle cells and pleomorphic cells stained positively for cyclin D1 (Fig. 2B), and the simultaneous expression of CD20 and cyclin D1 was demonstrated on serial sections (Fig. 2C, 2D). In both cases, the pleomorphic cells exhibited intense staining for PCNA, but cells showing weaker staining predominated in mantle cell areas (Fig. 2E).
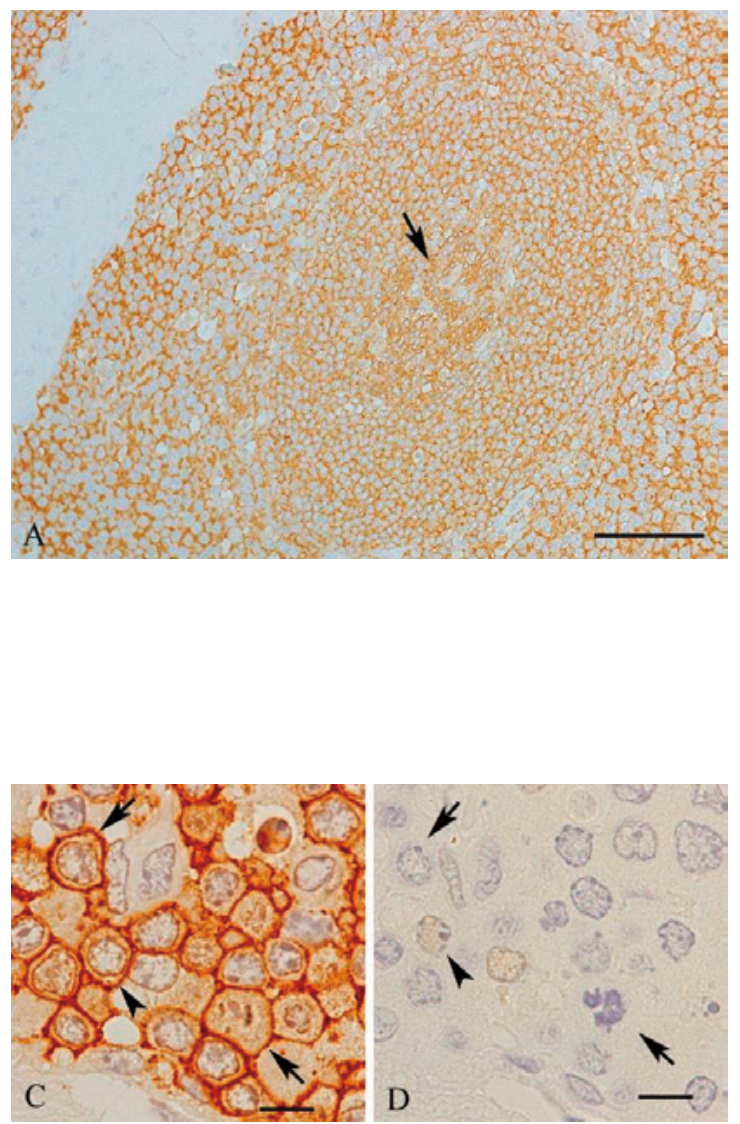

\section{Discussion}

Human mantle cell lymphoma is postulated to be of mantle zone lymphocyte origin, and may show a mantle zone growth pattern (Swerdlow et al. 2008). The lymphoma cells are usually positive for CD5, and nearly all cases overexpress cyclin D1 (Chan 2007). Such histological and immunophenotypic features were also seen in the present bovine cases, and a diagnosis of mantle cell lymphoma was made. The absence of immunoglobulin production supported this view (Feller \& Diebold 2004). Large and pleomorphic cells including cells with prominent nucleoli are observed in the pleomorphic variant of human mantle cell lymphoma (Swerdlow et al. 2008). In contrast, the

Fig. 2A. Case 1, lymph node of the paralumbar fossa

Lymphoma cells (neoplastic mantle cells and surrounding pleomorphic cells) and follicle center cells in a residual follicle center (arrow) are all positive for CD20. [SAB; Bar $=50 \mu \mathrm{m}]$

Fig. 2B. Case 1, spleen

Several neoplastic mantle cells with weak nuclear expression of cyclin D1 are visible in this field. [SAB; Bar $=10 \mu \mathrm{m}$ ]

Fig. 2C. Case 1, hepatic lymph node

The marginal sinus is filled with CD20-positive pleomorphic cells (arrows and arrowhead). [SAB; Bar $=5 \mu \mathrm{m}$ ]

Fig. 2D. Case 1, hepatic lymph node

Adjacent section shows the same cells as those depicted in Fig. 2C. Most pleomorphic cells are negative for cyclin D1 (arrows), but nuclear positivity is observed in a pleomorphic cell (arrowhead). [SAB; Bar $=5 \mu \mathrm{m}]$

Fig. 2E. Case 1, lymph node of the paralumbar fossa

Large pleomorphic cells stain intensely for PCNA, but most neoplastic mantle cells react weakly. [SAB; Bar $=50 \mu \mathrm{m}$ ] 
current cases showed histological transition from neoplastic mantle cells to larger and more pleomorphic cells, and the latter morphologically corresponded to pleomorphic B cell lymphoma cells (Murayama et al. 2011, Iwama et al. 2013, Hagiwara et al. 2014). Lymph nodes composed chiefly of neoplastic pleomorphic cells showed a tendency toward severe enlargement in case 1 , and thus were considered to be in the later stages of tumor development. The greater number of mitotic figures in pleomorphic cell areas indicates that focal mantle cell areas are entirely replaced by diffusely growing pleomorphic cells at the terminal stage. Likewise, in case 2, neoplastic mantle cells, which were more pleomorphic than in case 1 , presumably show a transition to a diffuse growth pattern at more advanced stages. In humans, the proliferation of mantle cell lymphoma initially develops in areas surrounding reactive germinal centers (mantle zone pattern), finally resulting in a diffuse pattern (Feller \& Diebold 2004).

A case of B-immunoblastic lymphoma evolving from a mantle cell lymphoma has been reported in humans, but the transition of mantle cell lymphoma to centroblastic lymphoma (diffuse cell) has not (Feller \& Diebold 2004). The presence of cIg may be observed in follicular or follicle center cell lymphomas and more mature cells in cattle and swine (Kadota \& Niibori 1985, Kadota et al. 1986, Kadota \& Nakajima 1988, Kameyama et al. 2003, Honda et al. 2009, Murayama et al. 2011, Nishijo et al. 2013, Hagiwara et al. 2014). The absence of $\mathrm{cIg}$ in human and bovine mantle cell lymphomas can be explained by the fact that mantle cells correspond to naive pre-germinal center B cells (Feller \& Diebold 2004). Pleomorphic B cell lymphoma is characterized by a lack of staining for cIg in cattle (Iwama et al. 2013); therefore, we suggest that neoplastic mantle cells in the present cases have transformed into more atypical and pleomorphic cells, but have not differentiated into more mature cells such as cIg-producing immunoblasts (Murayama et al. 2011).

Most BLV-associated lymphomas are classified as pleomorphic B cell lymphomas (Iwama et al. 2013, Hagiwara et al. 2014). On the basis of cytological atypia and pleomorphism in some areas, the current cases were judged to be associated with the virus (Iwama et al. 2013). Neoplastic transformation and progression in domestic animals are generally quite rapid, and it should be noted that the short-time transition to more malignant (dedifferentiated) or more mature histological types (Kadota \& Nakajima 1988) may complicate the histological diagnosis of malignant tumors, especially of lymphohematopoietic neoplasms. In addition, considerable differences relative to human cases (Ogihara et al. 2012) may cause difficulties in identifying the cytological features of bovine lymphoid neoplasms, and the expression of immunohistochemical markers is commonly diminished by neoplastic transformation and progression (Iwama et al. 2013).

In the present molecular study, the number of BLV copies was intermediate between a case of pleomorphic B cell lymphoma (18,059 copies/100 ng DNA and 8,037 copies in the superficial cervical and subiliac lymph nodes, respectively) (Kadota \& Ishikawa 2017a) and a case of acute basophilic leukemia (160 copies in the lateral iliac lymph node) (Kadota \& Ishikawa 2017b). Together with data from an early stage case of nodal pleomorphic lymphoma where the proliferation of both pleomorphic cells and neoplastic mantle cells was observed in some lymph nodes, with 240-5,994 copies/100 ng DNA being quantified (unpublished data), the copy number in BLV-associated mantle cell lymphoma may tend to be lower than in BLV-associated pleomorphic B cell lymphoma.

\section{References}

Abe, Y. et al. (2007) Immunohistochemical study of lymphomas of abdominal cavity origin in two cows with bovine leukemia virus. Jpn. Agric. Res. Quart., 41, 153-156.

Chan, C. (2007) Tumors of the lymphoreticular system. In Diagnostic Pathology of Tumors, 3rd ed., ed. Fletcher, C. D. M., Churchill Livingstone, London, 1139-1361.

Feller, A. C. \& Diebold, J. (2004) Mantle cell lymphoma. In Histopathology of Nodal and Extranodal Non-Hodgkin's Lymphomas, 3rd ed., Springer, Berlin, 66-75.

Hagiwara, A. et al. (2014) A histological study of lymphoid neoplasms in cattle infected with bovine leukosis virus. $J$. Jpn. Vet. Med. Assoc., 67, 199-203 [In Japanese with English summary].

Honda, H. et al. (2009) Lymphoplasmacytoid lymphoma in a cow. J. Vet. Med. Sci., 71, 673-676.

Iwama, Y. et al. (2013) Cytology of B cell lymphoma in cattle infected with bovine leukosis virus. Jpn. Agric. Res. Quart., 47, 103-107.

Kadota, K. et al. (1986) Three types of swine immunoglobulinproducing tumours: lymphoplasmacytic lymphosarcoma, immunoblastic lymphosarcoma and plasmacytoma. J. Comp. Pathol., 96, 541-550.

Kadota, K. \& Ishikawa, Y. (2017a) A proposal for the histological classification of B cell neoplasms in cattle. Bull. NARO Natl. Inst. Anim. Hlth., 123, 11-22 [In Japanese with English summary].

Kadota, K. \& Ishikawa, Y. (2017b) A proposal for the histological classification of myeloid neoplasms in cattle. Bull. NARO Natl. Inst. Anim. Hlth., 123, 35-46 [In Japanese with English summary].

Kadota, K. \& Nakajima, H. (1988) Histological progression of follicular centre cell lymphomas to immunoglobulin-producing tumours in two pigs. J. Comp. Pathol., 99, 145-158.

Kadota, K. \& Niibori, S. (1985) A case of swine follicular lymphoma with intracytoplasmic immunoglobulin inclusions. 


\section{J. Comp. Pathol., 95, 599-608.}

Kagawa, Y. et al. (2009) Immunohistochemical characterization of five types of lymphoid neoplasms in calves. Jpn. Agric. Res. Quart., 43, 239-245.

Kameyama, M. et al. (2003) Plasma cell myeloma producing IgG, IgM, and IgA immunoglobulins in a cow. J. Vet. Diagn. Invest., 15, 166-169.

Murayama, S. et al. (2011) Cytologic and immunophenotypic investigation of lymphohematopoietic neoplasms in cattle. Jpn. Agric. Res. Quart., 45, 225-231.

Nishijo, S. et al. (2013) Signet ring cell lymphoma with plasmacytic differentiation in a pig. J. Vet. Med. Sci., 75, 799-802.
Ogihara, K. et al. (2012) Lymphoid neoplasms in swine. J. Vet. Med. Sci., 74, 149-154.

Somura, Y. et al. (2014) Comparison of the copy numbers of bovine leukemia virus in the lymph nodes of cattle with enzootic bovine leukosis and cattle with latent infection. Arch. Virol., 159, 2693-2697.

Swerdlow, S. H. et al. (2008) Mantle cell lymphoma. In WHO Classification of Tumours of Haematopoietic and Lymphoid Tissues, 4th ed., eds. Swerdlow, S. H. et al., International Agency for Research on Cancer, Lyon, 229-232.

Valli, V. E. et al. (2006) Canine indolent nodular lymphoma. Vet. Pathol., 43, 241-256. 